\title{
Interactive comment on "Numerical simulations of glacier evolution performed using flow-line models of varying complexity" by Antonija Rimac et al.
}

\section{Anonymous Referee \#1}

Received and published: 26 April 2017

In this paper a comparison between the Shallow Ice Approximation (SIA) and a FullStokes Model (FSM) implemented in Elmer/lce is presented. Results from several test-cases are shown and discussed and some conclusions are drawn from these. However, not much theory is presented that supports the results obtained. The paper is well-written but lacks important references and reflections on how the current findings relate to the research front line.

What is perhaps the most problematic issue with this paper is that it lacks references to [1] and [2] that deal with comparisons between SIA, Second Order Shallow Ice Approximation (SOSIA) and FSM. Moreover, in these papers the validity of the assumptions behind SIA and SOSIA is thouroughly investigated, theoretically and numerically. Also, the importance of the aspect ratio $\mathrm{e}$ - the characteristic height of the ice divided by the characteristic length - was numerically and theoretically studied. In order to merit

Printer-friendly version

Discussion paper 
for publication, the authors must relate their results with respect to these papers. How much is actually new here and how much can be deduced from the results in [1] and [2]?

More detailed comments include:

- Page 3, line 19: At the...

- Presentation of bedrocks: please illustrate with one or more figures. As it is now it is difficult to understand the difference in the geometries of the simulations run in e.g. Sections 4.1 and 4.2 .

- Overall, the references are fairly old. Apart from [1] and [2] it should be possible to include more relevant and up-to-date references.

- In most figures there is a mistake in the caption between (b) and (c).

To summarize, my opinion is that if the authors rewrite the paper thoroughly in relation to [1] and [2], it might be acceptable for publication. However, that strongly depends on how much is found to be new in the present paper when this is included.

\section{References:}

[1] Josefin Ahlkrona, Nina Kirchner, Per Lötstedt; A numerical study of scaling relations for non-newtonian thin-film flows with applications in ice sheet modelling. $/ Q \mathrm{~J}$ Mechanics Appl Math/ 2013.

[2] Josefin Ahlkrona, Nina Kirchner, Per Lötstedt; Accuracy of the zeroth-and secondorder shallow-ice approximation-numerical and theoretical results. /Geoscientific Model Development/, 2013.

Printer-friendly version

Interactive comment on Geosci. Model Dev. Discuss., doi:10.5194/gmd-2017-67, 2017. 